\title{
Chemical Wave Logic Gates
}

\author{
Oliver Steinbock, ${ }^{\dagger}$ Petteri Kettunen, ${ }^{\ddagger}$ and Kenneth Showalter* \\ Department of Chemistry, West Virginia University, Morgantown, West Virginia 26506-6045
}

Received: April 29, 1996; In Final Form: August 6, $1996^{\otimes}$

\begin{abstract}
Logic gates based on chemical wave propagation in geometrically constrained excitable media are demonstrated in a Belousov-Zhabotinsky membrane system. The catalyst of the reaction is printed in specific predetermined patterns with geometries designed to provide various logic operations. Computational studies of the serial coupling of elements to form multicomponent gates and general chemical wave circuitry are presented.
\end{abstract}

\section{Introduction}

Propagating wave behavior is pervasive in living organisms, from waves of electrical activity in heart tissue ${ }^{1}$ to action potentials in neuronal systems., ${ }^{2,3}$ Waves in excitable media serve to transform information in the temporal domain, for example, the rhythm of pacemaker cells or the firing of a neuron, into signals in the spatial domain, which are transmitted from one location to another. In addition to signal transmission, propagating waves can provide information on optimal pathways through spatial networks and mazes, ${ }^{4,5}$ thus offering a mechanism for self-optimization in biological processes, such as the aggregation of amoebae in the slime mold Dictyostelium discoideum. ${ }^{6,7}$ Propagating waves can also provide the basis for information processing, such as image manipulation ${ }^{8}$ and simple computational tasks. ${ }^{9,10}$ Image edge enhancement, for example, relies on the transition of phase waves to autowaves, converting shallow gradients of an image into sharp boundaries. ${ }^{11,12}$ Logic gates that rely on wave initiation as a threshold switch have been based on waves propagating through narrow channels. ${ }^{9}$ Constant velocity wave propagation has even been used to measure the value of $\pi, \sqrt{ } 2$, and the golden mean. ${ }^{10}$ In this report, we describe a new scheme for carrying out logic operations with propagating chemical waves that is based on determining optimal pathways through specific geometrical configurations of excitable media.

A conceptual framework for chemical based computations has been advanced in a series of papers by Ross and coworkers. ${ }^{13-16}$ They proposed operational models for the experimental implementation of various logic gates and simple circuits based on coupled reactors with nonlinear chemical reactions. Hjelmfelt, Schneider, and Ross ${ }^{17}$ proposed a chemical neural network for pattern recognition, and Laplante et al. ${ }^{18}$ have implemented such a network in an experimental system consisting of bistable iodate-arsenite reactions in eight coupled CSTRs. Other studies of logic gates using bistable reactions have been carried out by Schneider et al., ${ }^{19,20}$ and enzymatic reactions as switches in reaction networks have been considered by Okamoto and co-workers. ${ }^{21}$ A recent study by Adleman ${ }^{22}$ has shown how the manipulation of DNA sequences can be used to solve directed Hamiltonian path problems. Perhaps the earliest suggestion of chemical-based computation was by Rössler, ${ }^{23}$ who, some 20 years ago, proposed using bistability as a switch in logic devices.

\footnotetext{
* To whom correspondence should be addressed.

$\uparrow$ Permanent address: Institut für Experimentelle Physik, Otto-vonGuericke-Universität, Universitätsplatz 2, D-39106 Magdeburg, Germany.

\# Permanent address: Department of Physical Sciences, Division of Biophysics, University of Oulu, Linnanmaa, FIN-90570 Oulu, Finland.

${ }^{\otimes}$ Abstract published in Advance ACS Abstracts, November 1, 1996.
}

Following Ross and co-workers, ${ }^{13-16}$ a scheme for logic gates based on the propagation of chemical waves through narrow channels with no-flux boundaries has been developed. ${ }^{9}$ Excitable Belousov-Zhabotinsky ${ }^{24}$ (BZ) reaction mixtures were connected by precision-bore capillary tubes through which waves could propagate from one compartment to another. When the tube diameter is greater than a critical value, the wave propagating through the tube serves to initiate a wave in the second compartment; otherwise, the hemisphere of excited solution at the tube exit collapses and no wave is initiated. ${ }^{25-27}$ Two or more tubes can be configured to form logic gates, which rely on the union of subcritical regions of excitation to give rise to wave initiation. The geometrical configuration, the spatiotemporal synchronization of the waves, and the ratio of the tube radius to the critical radius of the excitable medium determine the features of the gates.

In this paper, we study chemical wave circuity prepared by printing the catalyst of the $\mathrm{BZ}$ reaction in predetermined patterns onto a membrane support. ${ }^{28}$ Chemical waves propagate with constant velocity and, provided that curvature effects are negligible, ${ }^{29}$ advance according to Huygens's principle. We use these basic properties of chemical waves in geometrically constrained excitable media to develop gates that yield logic operations between input stimuli and output responses.

The chemical wave circuitry is generated by printing bathoferroin, a catalyst for the $\mathrm{BZ}$ reaction, ${ }^{30}$ on polysulfone membranes with an ink-jet printer. ${ }^{28}$ Following Noszticzius and co-workers, ${ }^{31}$ the patterned membrane is placed on an agarose gel containing a catalyst-free $\mathrm{BZ}$ reaction mixture. The gelmembrane configuration provides a quasi-open, reactiondiffusion system that is free from convective disturbances and spontaneous pacemakers. Waves can be reproducibly initiated at desired locations by contacting a silver wire to the catalystloaded membrane surface. The geometry of a particular gate determines the nature of its operation, which can be modified during the experiment by suitable external perturbations.

\section{Experimental Procedures}

A $5 \mathrm{mM}$ bathoferroin [tris(4,7-diphenyl-1,10-phenanthroline)iron(II)] solution (in glacial acetic acid) was printed on polysulfone membranes ${ }^{31}$ (Gelman Supor-450) using an inkjet printer (Hewlett Packard DeskJet 520, HP51626A cartridge). The membrane was supported on agarose gel $(1.5 \% \mathrm{w} / \mathrm{v})$ containing $0.22 \mathrm{M} \mathrm{NaBrO}_{3}, 0.27 \mathrm{M} \mathrm{H}_{2} \mathrm{SO}_{4}, 0.17 \mathrm{M}$ malonic acid, and $0.1 \mathrm{M} \mathrm{NaBr}$ (prior to bromination of malonic acid). The temperature was maintained at $25 \pm 1{ }^{\circ} \mathrm{C}$.

A CCD video camera was used to monitor the experiment, measuring gray levels of monochromatic light $(\lambda=500 \mathrm{~nm})$ reflected from the printed membrane. Images showing wave 
(a)

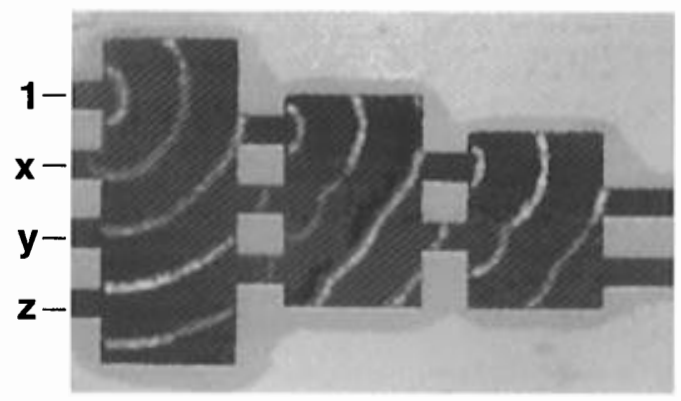

(b)

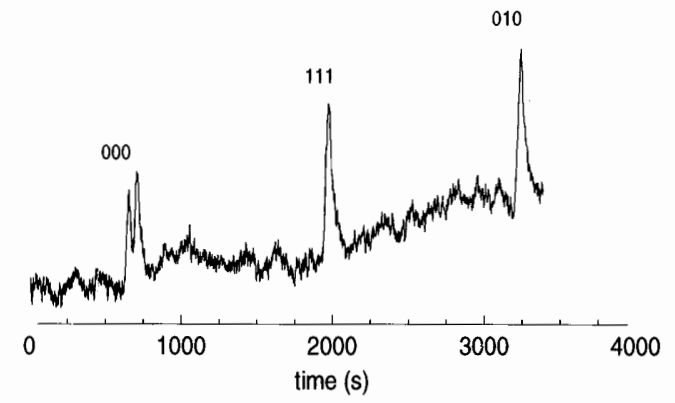

Figure 1. (a) Three-layer chemical wave network representing an $\mathrm{OR}(\mathrm{x}, \mathrm{y}, \mathrm{z})$ gate. Wave evolution for the input vector $(\mathbf{0 , 0 , 0})$ is shown as superimposed snapshots at intervals of $\Delta t$. Image area and $\Delta t: 6.4$ $\mathrm{cm}^{2}, 110 \mathrm{~s}$. (b) Gray-level intensity signals at output channels in response to three different inputs, $(\mathbf{x}, \mathbf{y}, \mathbf{z})=\{(\mathbf{0}, \mathbf{0}, \mathbf{0}),(\mathbf{1}, \mathbf{1}, \mathbf{1}),(\mathbf{0}, \mathbf{1}, \mathbf{0})\}$. Single and double peaks correspond to True and False, respectively. (The increasing intensity of the base line reflects the aging of the system.)

position at successive time increments ( $\Delta t$ in figure captions) were generated by overlaying difference images onto the image of the printed membrane. Each difference image was obtained by subtracting successive images separated by a $10 \mathrm{~s}$ interval.

\section{Experimental Results}

Figure 1a shows an example of a chemical wave circuit with a geometry corresponding to an $\mathrm{OR}(\mathrm{x}, \mathrm{y}, \mathrm{z})$ gate. Catalyst-loaded domains appear as dark rectangular regions on the background of the surrounding catalyst-free membrane. The gate was constructed with four initiation sites on the left (labeled 1, $\mathbf{x}, \mathbf{y}$, $\mathbf{z})$ and two output channels on the right. The input $(\mathbf{x}, \mathbf{y}, \mathbf{z})$ is defined by the presence or absence of wave initiation in each of the channels at a specified time, corresponding to True (1) or False (0). An additional "clock" input, a wave simultaneously initiated at input $\mathbf{1}$, is required by the gate to establish the output for the case of zero input, i.e., $(\mathbf{x}, \mathbf{y}, \mathbf{z})=(\mathbf{0 , 0 , 0})$. The output is defined as True or False, respectively, by synchronous or asynchronous waves exiting the two channels on the right. The output was monitored as a function of time by summing the gray-level intensities of a $5 \times 5$ pixel area $\left(0.2 \mathrm{~mm}^{2}\right)$ in each of the two output channels. In this representation, the values True or False correspond to single- or double-maximum signals. The time series in Figure 1b shows output signals of the gate corresponding to the inputs indicated above each response.

The operation of the $\operatorname{OR}(\mathrm{x}, \mathrm{y}, \mathrm{z})$ gate is illustrated by superimposing snapshots of a wave originating from input channel 1, shown in Figure 1a. In this example, the input $(\mathbf{x}, \mathbf{y}, \mathbf{z})=$ $(\mathbf{0 , 0 , 0})$ gives rise to asynchronous waves at the output channels corresponding to an output of False (0), as shown in Figure 1b. Each of the other seven input possibilities gives rise to synchronous waves at the output channels, two of which, $(\mathbf{1}, \mathbf{1}, \mathbf{1})$ and $(\mathbf{0 , 1 , 0})$, are illustrated in Figure 1b. These inputs correspond to outputs of True (1). (a)

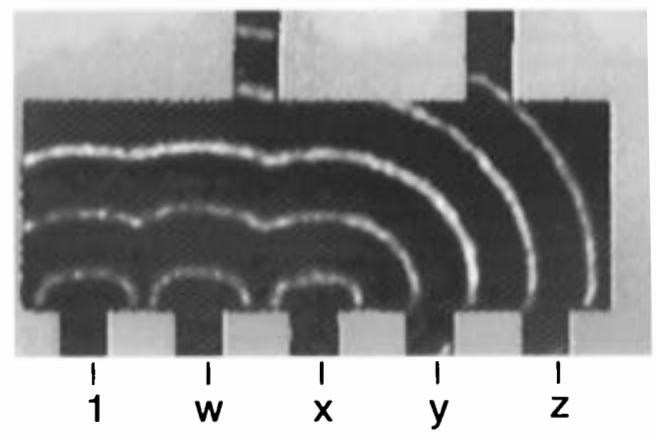

(b)

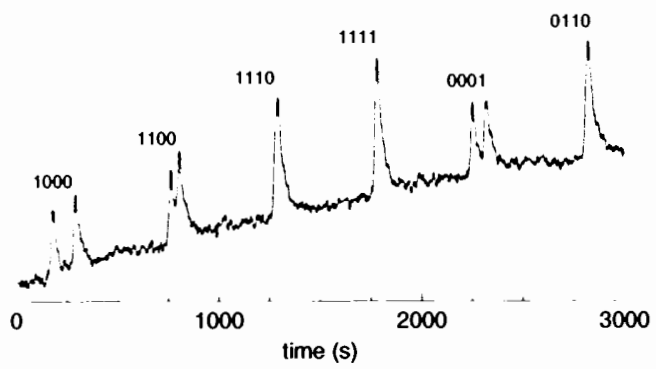

Figure 2. (a) One-layer chemical wave network representing an $\operatorname{AND}[\mathrm{OR}(\mathrm{w}, \mathrm{x}), \mathrm{OR}(\mathrm{y}, \mathrm{z})]$ gate. Wave evolution for input $(\mathbf{1 , 1 , 0 , 0})$ is shown as superimposed snapshots at intervals of $\Delta t$. Image area and $\Delta t: 2.5 \mathrm{~cm}^{2}, 50 \mathrm{~s}$. (b) Output signals in response to six different inputs, $(\mathbf{w}, \mathbf{x}, \mathbf{y}, \mathbf{z})=\{(\mathbf{1}, \mathbf{0}, \mathbf{0}, \mathbf{0}),(\mathbf{1}, \mathbf{1}, \mathbf{0}, \mathbf{0}),(\mathbf{1}, \mathbf{1}, \mathbf{1}, \mathbf{0}),(\mathbf{1}, \mathbf{1}, \mathbf{1}, \mathbf{1}),(\mathbf{0}, \mathbf{0}, \mathbf{0}, \mathbf{1}),(\mathbf{0}, \mathbf{1}, \mathbf{1}, \mathbf{0})\}$.

The realization of logic gates from geometrically constrained excitable media is not limited to simple OR operations. Figure 2a shows a configuration that yields a gate corresponding to $\operatorname{AND}[\mathrm{OR}(\mathrm{w}, \mathrm{x}), \mathrm{OR}(\mathrm{y}, \mathrm{z})]$ for the 16 possible input combinations of $(\mathbf{w}, \mathbf{x}, \mathbf{y}, \mathbf{z})$. The superimposed snapshots illustrate the input $(\mathbf{1}, \mathbf{1}, \mathbf{0 , 0})$. The OR of $\mathbf{y}$ and $\mathbf{z}$ for this input vector is False, and the corresponding output is therefore False, with asynchronous waves at the output channels. The time series in Figure $2 b$ shows examples of six output signals corresponding to the indicated input vectors.

Even very simple geometries can give rise to complex logic operations, such as the $\mathrm{OR}[\mathrm{z}, \mathrm{AND}[\mathrm{x}, \mathrm{NOT}(\mathrm{y})]]$ gate shown in Figure 3. Because input $\mathbf{z}$ has equal-length connections to the output channels, the output is always True if a wave is initiated in $\mathbf{z}$. Of the four possible cases where $\mathbf{z}$ is False, only one combination can generate symmetric excitations at the output channels. This combination is given by $(\mathbf{x}, \mathbf{y}, \mathbf{z})=(\mathbf{1}, \mathbf{0}, \mathbf{0})$ and corresponds to the wave evolution shown in Figure 3a. A time series illustrating the symmetrical signal for this combination, together with example signals for five other combinations, is shown in Figure 3b.

Many additional gates can be devised, which, like the examples above, have functions determined by the geometry of the catalyst-loaded domains. This approach can also be extended to gates capable of solving varying tasks. The function of a gate can be significantly altered by opening and closing strategically located pathways in the excitable network. Figure 4 shows a system in which a small platinum electrode was placed beneath the central door connecting the two rectangular domains. Wave propagation in the door area was readily suppressed by applying a negative potential to the electrode for $\sim 15 \mathrm{~s}$. The geometry of the gate with the closed channel yields True outputs for $(\mathbf{x}, \mathbf{y}, \mathbf{z}) \in\{(\mathbf{0}, \mathbf{0}, \mathbf{0}),(\mathbf{1}, \mathbf{0}, \mathbf{1}),(\mathbf{0}, \mathbf{1}, \mathbf{0})$, $(\mathbf{1}, \mathbf{1}, \mathbf{1})\}$, thus providing pattern recognition of the symmetric input combinations. Figure $4 \mathrm{a}$ shows the wave evolution for $(\mathbf{x}, \mathbf{y}, \mathbf{z})=(\mathbf{1}, \mathbf{1}, \mathbf{0})$, and shown in Figure $4 \mathrm{~b}$ are output signals resulting from two symmetric and two asymmetric input combinations. 
(a)

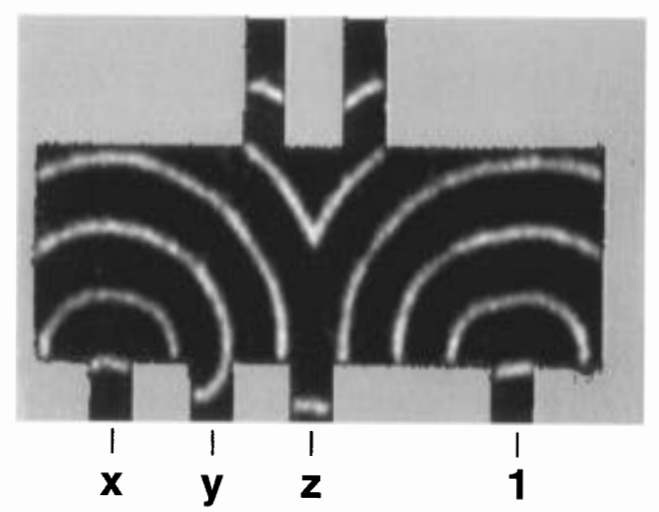

(b)

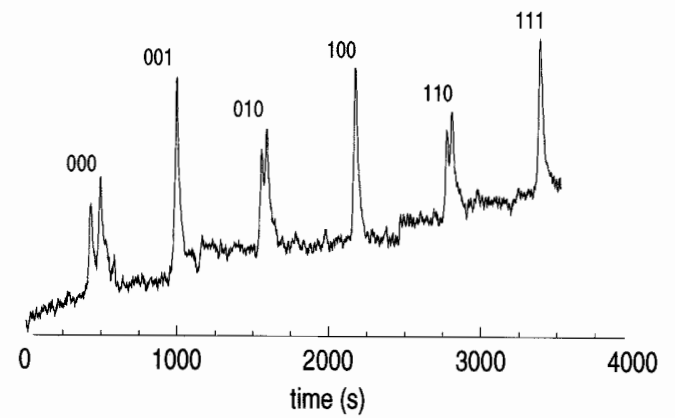

Figure 3. (a) One-layer chemical wave network representing an $\mathrm{OR}[\mathrm{z}, \mathrm{AND}[\mathrm{x}, \mathrm{NOT}(\mathrm{y})]]$ gate. The superimposed snapshots at intervals of $\Delta t$ show wave evolution for input $(\mathbf{1 , 0 , 0})$. Image area and $\Delta t: 3.2$ $\mathrm{cm}^{2}, 50 \mathrm{~s}$. Note that waves in input channels $\mathbf{y}$ and $\mathbf{z}$ arise from wave activity in the central rectangular domain and do not represent input waves. (b) Output signals in response to six different inputs, $(\mathbf{x}, \mathbf{y}, \mathbf{z})=$ $\{(\mathbf{0 , 0}, \mathbf{0}),(\mathbf{0}, \mathbf{0}, \mathbf{1}),(\mathbf{0}, \mathbf{1}, \mathbf{0}),(\mathbf{1}, \mathbf{0}, \mathbf{0}),(\mathbf{1}, \mathbf{1}, \mathbf{0}),(\mathbf{1}, \mathbf{1}, \mathbf{1})\}$.

The electrode-induced inhibition of wave propagation is reversible; however, a period of $\sim 2$ min following application of the potential must elapse before a wave can again propagate through the channel. This suggests that the applied potential results in an electrochemical reaction that inhibits wave propagation by lowering the local excitability of the medium. Diffusion of fresh reactants into the region provides a recovery mechanism such that the original composition is restored and wave propagation through the channel can again occur. Figure 5 shows the behavior when the door is open; the gate now yields True outputs for the additional input triplets $(\mathbf{1 , 1 , 0})$ and $(\mathbf{0 , 1}, \mathbf{1})$. The wave evolution for the input $(\mathbf{1 , 1 , 0})$ is shown in Figure 5a, and examples of output signals are shown in Figure $5 \mathrm{~b}$.

\section{Modeling Results}

In this section, we present a numerical study that addresses how the geometric scheme can be modified to enable the coupling of individual logic elements to form multicomponent gates. The modification involves changing the output from synchronous (asynchronous) signals to the presence (absence) of a signal for representing True (False). Such a modification is essential for the generality of the scheme since certain gates require serially linked components. It also provides a means for linking gates in the construction of complex circuitry.

We will consider an XOR gate comprised of an XNOR gate coupled to a NOT gate to illustrate the assembly of multicomponent gates. An $\mathrm{XNOR}(\mathrm{x}, \mathrm{z})$ gate can be created from the configuration shown in Figure $4 \mathrm{a}$ in which the $\mathbf{y}$ input channel is omitted; a NOT(x) gate can be created from the same configuration in which both the $\mathbf{y}$ and $\mathbf{z}$ input channels are omitted. When coupled, these gates function as an XOR gate if the output from the XNOR component becomes the input of (a)

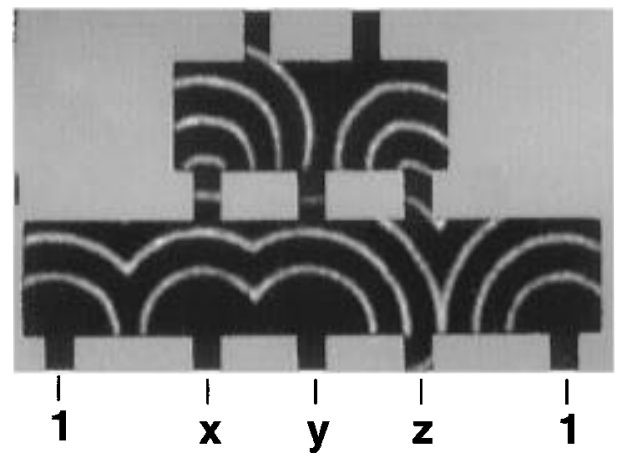

(b)

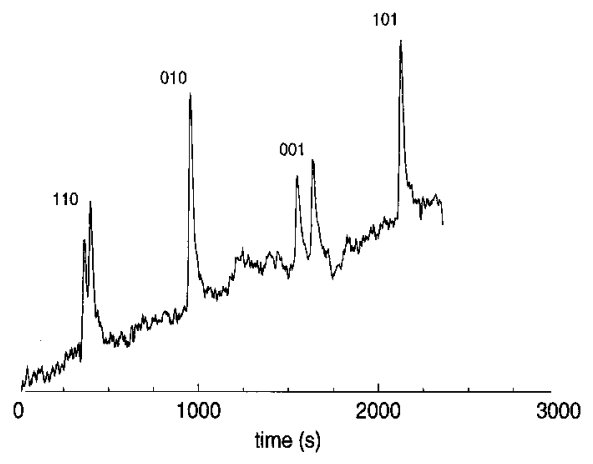

Figure 4. (a) Two-layer network with electrode inhibition of the central door corresponding to an $\mathrm{XNOR}(\mathrm{x}, \mathrm{z})$ gate. The superimposed snapshots show wave evolution for input $(\mathbf{1 , 1 , 0})$, which results in asynchronous excitations in the output channels (False). Image area and $\Delta t: 4.3 \mathrm{~cm}^{2}$, 40 s. (b) Time series showing output signals for inputs $(\mathbf{x}, \mathbf{y}, \mathbf{z})=$ $\{(\mathbf{1}, \mathbf{1}, \mathbf{0}),(\mathbf{0}, \mathbf{1}, \mathbf{0}),(\mathbf{0 , 0}, \mathbf{1}),(\mathbf{1}, \mathbf{0}, \mathbf{1})\}$. The voltage $(\sim 5 \mathrm{~V})$ and current $(\sim 8$ $\mathrm{mA}$ ) required to close the central door indicate that an electrode reaction takes place which temporarily reduces the local excitability of the medium. The reference electrode was positioned outside the observation area $\sim 2.4 \mathrm{~cm}$ from the Pt electrode.

the NOT component and this input is the presence or absence of a signal for True or False.

Our modeling study was carried out with a modified TysonFife $^{32,33}$ reduction of the Oregonator ${ }^{34}$ (see ref 28):

$$
\begin{gathered}
\frac{\partial u}{\partial \tau}=\nabla^{2} u+\frac{1}{\epsilon}\left[c\left(u-u^{2}-f v \frac{u-q}{u+q}\right)+(c-1) u^{2}\right] \\
\frac{\partial v}{\partial \tau}=c(u-v)
\end{gathered}
$$

where the variables $u$ and $v$ represent bromous acid and ferriin, and $c=1$ or 0 for catalyst-loaded or catalyst-free regions, respectively. No Laplacian term is included in eq 2 since both forms of the catalyst, ferroin and ferriin, are immobilized on the membrane surface. Because bromous acid can diffuse beyond the ferroin-loaded domains into the surrounding catalystfree regions, boundaries allowing the diffusive flux of $u$ were utilized. In the catalyst-free regions, the reaction is modeled by the term $(-1 / \epsilon)\left(u^{2}\right)$ in eq 1 , which describes the bimolecular disproportionation reaction of bromous acid. This modification was found to be necessary in earlier studies of wave behavior in catalyst-loaded domains separated by catalyst-free regions. ${ }^{28}$ In calculations where the disproportionation reaction was omitted, bromous acid accumulated to high concentrations in the catalyst-free regions.

We now consider how the output of each logic element can be modified to provide an on/off switch for True/False responses. This can be accomplished by creating narrow, 
(a)

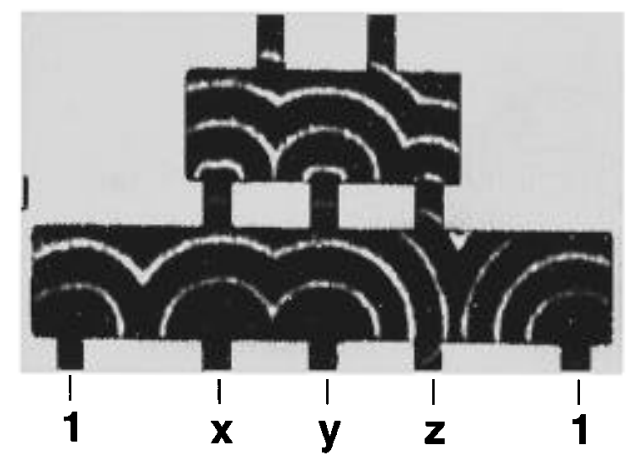

(b)

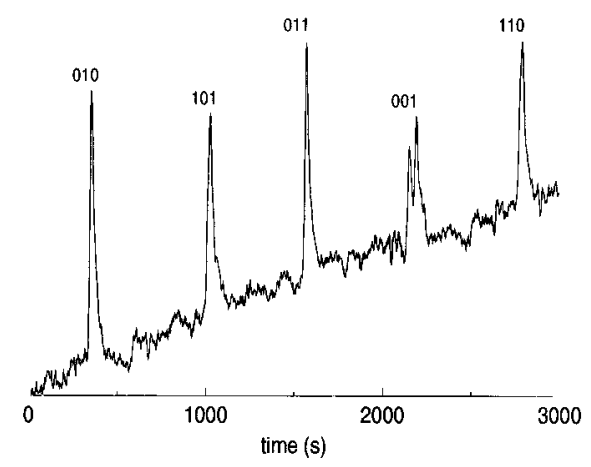

Figure 5. (a) Two-layer network as in Figure 4 without electrode inhibition of the central door. The gate switches from $\operatorname{XNOR}(x, z)$ to $\mathrm{OR}[\mathrm{y}, \mathrm{XNOR}(\mathrm{x}, \mathrm{z})]$ upon opening the door. The superimposed snapshots show wave evolution for input $(\mathbf{1 , 1 , 0})$, which results in synchronous excitations in the output channels (True). Image area and $\Delta t: 4.2 \mathrm{~cm}^{2}$, 40 s. (b) Time series showing output signals for inputs $(\mathbf{x}, \mathbf{y}, \mathbf{z})=$ $\{(\mathbf{0}, \mathbf{1}, \mathbf{0}),(\mathbf{1}, \mathbf{0}, \mathbf{1}),(\mathbf{0}, \mathbf{1}, \mathbf{1}),(\mathbf{0}, \mathbf{0}, \mathbf{1}),(\mathbf{1}, \mathbf{1}, \mathbf{0})\}$.

parallel output channels that are separated by a narrow gap. For a particular medium excitability, single waves fail to propagate in the channel for channel widths narrower than some critical value. This behavior is illustrated in Figure 6a, which shows a superimposed sequence of snapshots of the evolution and propagation failure of a single wave in the upper channel. A planar wave, initiated in the left compartment, enters the channel and propagates to about half its length before failing. For this particular channel width and medium excitability, the diffusive loss of bromous acid to the surrounding catalyst-free region is too great for the reaction in the ferroin-loaded channel to sustain wave propagation. A detailed characterization of the parameter dependence of the propagation failure has not been carried out; however, for the parameter values used in these calculations, failure occurs below a threshold channel width between 20 and 22 grid points (1.83 and 2.02 space units).

Figure $6 \mathrm{~b}$ shows the evolution of two waves propagating sideby-side for the same parameter values and channel width, where the channel separation is $2 / 5$ the channel width. Now, propagation failure does not occur because the waves reinforce each other, with the diffusion of bromous acid into the catalyst-free region between the channels serving to offset the diffusive loss. Significant accumulation of bromous acid develops in the gap between the aligned wave fronts, and this zone of elevated autocatalyst concentration travels along with the waves as they propagate from left to right. For the values of $\epsilon, f$, and $q$ and the channel width used here, the critical channel separation is on the order of $1 / 2$ the channel width for sustained propagation of aligned wave fronts. This mutual reinforcement to sustain wave propagation can also be seen for waves that are not precisely aligned. Figure $6 \mathrm{c}$ shows the behavior of two such waves, where the planar wave for the lower channel was initiated 0.16 time units after the initiation of the wave for the upper (a)

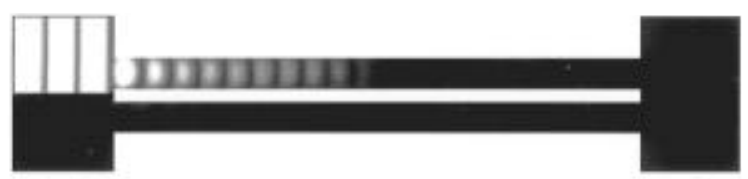

(b)

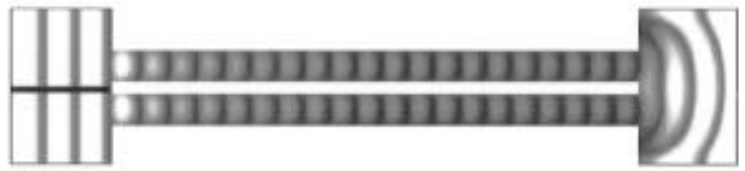

(c)

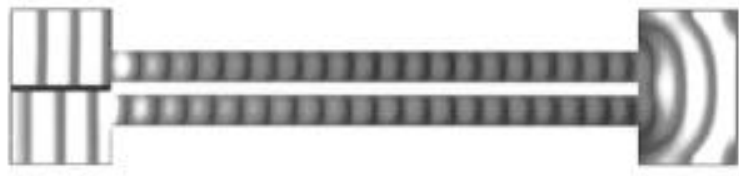

(d)

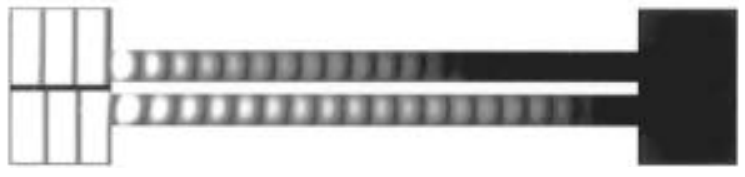

Figure 6. Wave evolution in narrow parallel channels calculated from eqs 1 and 2. Maximum and minimum concentrations of $u$ indicated by white and black, respectively. Solid line in left compartment indicates no-flux boundary separating input waves. (a) Propagation failure of single wave initiated in upper channel. (b) Propagation in upper and lower channels of aligned waves from initiation compartment on left to output compartment on right. (c) Propagation in upper and lower channels of waves to output compartment on right; wave in lower channel initiated 0.16 time units after wave was initiated in upper channel. (d) Propagation failure of waves in upper and lower channels; wave in lower channel initiated 0.32 time units after wave was initiated in upper channel. Calculations were carried out using the explicit Euler method with the Laplacian for bromous acid approximated by a fivepoint formula in eq 1 and the parameters $f=3.6, q=0.002$, and $\epsilon=$ 0.0585. Grid size $=464 \times 100, \Delta x=0.0917, \Delta \tau=3.50 \times 10^{-4}$, channel width $=20 \Delta x=1.83$, channel length $=336 \Delta x=30.8$, gap width $=8 \Delta x=0.733$, snapshot interval $=1000 \Delta \tau=0.350$.

channel. Again, the zone of elevated bromous acid in the gap is sufficient to sustain the wave propagation. Interestingly, the communication across the gap also serves to bring the wave fronts into alignment, as can be seen as the waves enter the output chamber on the right.

Figure 6d shows how propagation failure occurs when the difference in initiation times becomes too large, in this case 0.32 time units. The diffusive loss of bromous acid is now too large for sustained wave propagation. The effect of the bromous acid accumulation can still be seen, however, with the second wave (in the lower channel) propagating somewhat farther than the first wave. Calculations were carried out to determine an approximate value for the critical difference in initiation times above which propagation failure occurs. This value was found to be between 0.23 and 0.25 time units.

The behavior in Figure 6 can be used to provide an on/off switch for True/False responses in the geometric scheme, since symmetrical outputs give rise to aligned wave fronts and sustained wave propagation, while sufficiently unsymmetrical outputs lead to propagation failure. Figure 7 shows how the geometric scheme and the on/off switch based on parallel channel outputs can be combined. An XNOR(x,z) gate, similar to the configuration shown in Figure 4a except without an active $\mathbf{y}$ input channel, is modified to include narrow output channels. The wave evolution for the input $(\mathbf{x}, \mathbf{z})=(\mathbf{1}, \mathbf{1})$ is illustrated in Figure $7 \mathrm{a}$ as a sequence of superimposed snapshots. The symmetrical output of the gate gives rise to aligned waves in the parallel output channels, which propagate unabated to the final output domain (rectangular region on the right). Figure $7 \mathrm{~b}$ shows the wave evolution for the input $(\mathbf{x}, \mathbf{z})=(\mathbf{0 , 1})$, where, now, the unsymmetrical output results in propagation failure. 
(a)

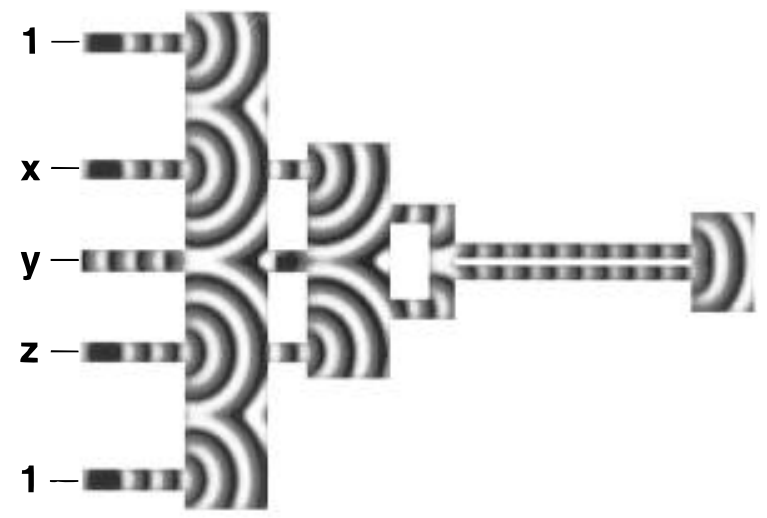

(b)

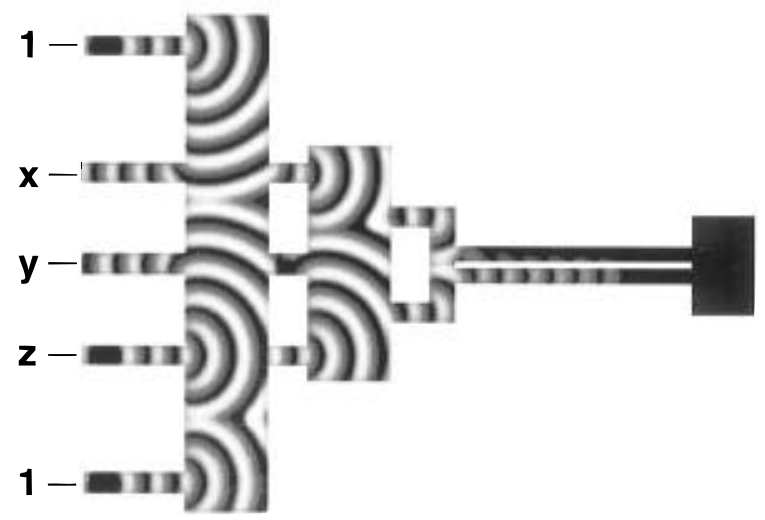

Figure 7. Modified geometric $\mathrm{XNOR}(\mathrm{x}, \mathrm{z})$ gate with parallel channel switch calculated from eqs 1 and 2. (a) Wave evolution for input (x,z) $=(\mathbf{1}, \mathbf{1})$ showing wave activity in output compartment for response of True. Note that waves in channel $\mathbf{y}$ arise from wave activity in the rectangular domain and do not represent input waves. (b) Evolution for input $(\mathbf{x}, \mathbf{z})=(\mathbf{0}, \mathbf{1})$ showing no wave activity in output compartment for response of False. Waves in channels $\mathbf{x}$ and $\mathbf{y}$ arise from wave activity in the rectangular domain and do not represent input waves. Wave propagation through central door connecting two rectangular domains was suppressed by setting variable $u$ to steady state value. Grid size $=450 \times 336, \Delta x=0.183, \Delta \tau=2.80 \times 10^{-3}$, channel width $=10 \Delta x=1.83$, channel length $=150 \Delta x=27.5$, gap width $=4 \Delta x=$ 0.733 , snapshot interval $=250 \Delta \tau=0.700$.

The success of the parallel channel switch depends on the output for negative responses to be sufficiently unsynchronized, which can be controlled by appropriate scaling of the geometrical scheme.

The configuration in Figure 7 becomes a NOT(x) gate when the $\mathbf{y}$ and $\mathbf{z}$ input channels are both deactivated. Thus, an $\mathrm{XOR}(\mathrm{x}, \mathrm{y})$ gate can be constructed by routing the output of the $\mathrm{XNOR}(\mathrm{x}, \mathrm{z})$ gate to the input of the NOT(x) gate. We note that the XOR gate is necessary for the generality of the scheme since it is required for important operations such as binary addition. Many other gates can be constructed by serially combining two or more existing gates. For example, an AND gate followed by a NOT gate becomes a NAND gate; an OR gate followed by a NOT gate becomes a NOR gate. The parallel channel switch provides the linking necessary for the construction of multicomponent gates as well as complex multiple-element circuits.

\section{Discussion and Conclusions}

The results presented here demonstrate that wave propagation in excitable media, with appropriate geometric constraints, can be used to perform computational tasks. The geometric logic elements are simple and robust, making them attractive as primary circuits for use in conjunction with additional elements such as threshold switches. Additional versatility in control of the logic functions can be realized with external perturbations by electrode reactions or, alternatively, by light irradiation in photosensitive systems. ${ }^{8,35}$

Composite gates made up of two or more coupled elements can be constructed to yield a wide variety of logic functions. This coupling can be implemented in the geometric scheme with the parallel channel switch. The ability to couple gates is necessary for the construction of general chemical wave circuitry, where both serial and parallel coupling can be envisioned. We note that threshold behavior such as the parallel channel switch can also serve as the basis for logic gates. It is possible to devise configurations based on this switch that represent all of the basic logic gates in a manner similar to the earlier scheme based on waves propagating through microcapillary tubes. ${ }^{9}$ However, the geometric scheme offers significant advantages over schemes based on threshold behavior. Relatively simple configurations lead to complex logic operations, unlike threshold gates, which require increasingly intricate configurations. The geometric scheme is also much more robust, since it depends only on constant velocity wave propagation. For certain operations, however, such as the XOR gate, it is necessary to embellish this scheme by employing some form of threshold switch.

The modeling study was carried out to determine if an on/ off switch could be developed that would make the geometric scheme more general by allowing the construction of multicomponent gates and complex chemical wave circuitry. We believe that the qualitative features of the parallel channel switch represent "proof of principle" that this type of on/off element is feasible. Studies using a more complete model may be required, however, for an accurate reflection of the anticipated experimental behavior. This is suggested by the wave propagation failure in Figure 6d, revealing how each wave responds to the accumulation of bromous acid in the gap separating the channels. In an experimental system we would expect that bromide ion, generated in the wake of the first wave, would tend to inhibit the advance of the second wave. Thus, we anticipate that the order in which the waves fail would reverse in more refined calculations where the diffusion of bromide ion is taken into account. Preliminary experiments on the parallel channel switch indicate that a higher resolution in the catalyst printing will be required than that used in our experimental studies to date. ${ }^{28}$

What is the purpose of demonstrating the feasibility of chemical-based computational devices? In comparison with electronic-based computations, chemical computations occur on time scales that are orders of magnitude longer and require space scales that are orders of magnitude larger. There can be only one reason for constructing computational devices based on excitable chemical media: to determine what such chemical systems can and cannot do. In so doing, we explore the possibilities for information processing by all excitable media, chemical and biological, with the chance of garnering insights into the workings of information processing in living systems.

Acknowledgment. We dedicate this paper to John Ross. K.S. thanks the National Science Foundation (CHE-9531515), the Office of Naval Research, and the Petroleum Research Fund for supporting this research. O.S. thanks the "Fonds der Chemischen Industrie" for a Liebig Fellowship. 


\section{References and Notes}

(1) Winfree, A. T. The Geometry of Biological Time; Springer: Berlin, 1980. Winfree, A. T. When Time Breaks Down; Princeton University Press: Princeton, 1987.

(2) Hall, Z. W., Ed. Molecular Neurobiology; Sinauer Pub.: Sunderland, MA, 1992.

(3) Murray, J. D. Mathematical Biology; Springer: Berlin, 1989.

(4) Sepulchre, J. A.; Babloyantz, A., Steels, L. In Proceedings of the International Conference on Artificial Neural Networks; Kohonen, T., Makisara, K., Simula, O., Eds.; Elsevier: Amsterdam, 1991; pp 12651268. Sepulchre, J. A.; Babloyantz, A. In Chemical Waves and Patterns; Kapral, R., Showalter, K., Eds.; Kluwer: Dordrecht, 1995; pp 191-217.

(5) Steinbock, O.; Tóth, Á.; Showalter, K. Science 1995, 267, 868.

(6) Devreotes, P. N. Science 1989, 245, 1054.

(7) Steinbock, O.; Showalter, K. Science 1995, 269, 418.

(8) Kuhnert, L.; Agladze, K. I.; Krinsky, V. I. Nature 1989, 337, 244.

(9) Tóth, Á.; Showalter, K. J. Chem. Phys. 1995, 103, 2058.

(10) Steinbock, O.; Kettunen, P. Chem. Phys. Lett. 1996, 251, 305.

(11) Aliev, R. R. J. Phys. Chem. 1994, 98, 3999.

(12) Yamaguchi, T.; Amemiya, T.; Nakaiwa, M.; Ohmori, T. Chem. Phys. Lett., submitted.

(13) Hjelmfelt, A.; Weinberger, E. D.; Ross, J. Proc. Natl. Acad. Sci. U.S.A. 1991, 88, 10983.

(14) Hjelmfelt, A.; Weinberger, E. D.; Ross, J. Proc. Natl. Acad. Sci. U.S.A. 1992, 89, 383.

(15) Hjelmfelt, A.; Ross, J. Proc. Natl. Acad. Sci. U.S.A. 1992, 89, 388.

(16) Hjelmfelt, A.; Ross, J. Proc. Natl. Acad. Sci. U.S.A. 1994, 91, 63.

(17) Hjelmfelt, A.; Schneider, F. W.; Ross, J. Science 1993, 260, 335.
(18) Laplante, J.-P.; Pumberton, M.; Hjelmfelt, A.; Ross, J. J. Phys. Chem. 1995, 99, 10063.

(19) Lebender, D.; Schneider, F. W. J. Phys. Chem. 1994, 98, 7533.

(20) Zeyer, K.-P.; Dechert, G.; Hohmann, W.; Blittersdorf, R.; Schneider, F. W. Z. Naturforsch. 1994, 49a, 953.

(21) Okamoto, M.; Sakai, T.; Hayashi, K. Biol. Cybern. 1988, 58, 295.

(22) Adleman, L. M. Science 1994, 226, 1021.

(23) Rössler, O. E. In Physics and Mathematics of the Nervous System; Conrad, M., Güttinger, W., Dal Cin, M., Eds.; Springer-Verlag: Berlin, 1974; pp 399-418.

(24) Zaikin, A. N.; Zhabotinsky, A. M. Nature 1970, 225, 535.

(25) Babloyantz, A.; Sepulchre, J. A. Physica D 1991, 49, 52.

(26) Babloyantz, A.; Sepulchre, J. A. Phys. Rev. Lett. 1991, 66, 1314

(27) Tóth, Á.; Gáspár, V.; Showalter, K. J. Phys. Chem. 1994, 98, 522.

(28) Steinbock, O.; Kettunen, P.; Showalter, K. Science 1995, 269, 1857.

(29) Tyson, J. J.; Keener, J. P. Physica D 1988, 32, 327. Zykov, V. S. Biophysics 1980, 25, 906.

(30) Yamaguchi, T.; Kuhnert, L.; Nagy-Ungvarai, Zs.; Müller, S. C.; Hess, B. J. Phys. Chem. 1991, 95, 5831.

(31) Lázár, A.; Noszticzius, Z.; Farkas, H.; Försterling, H.-D. Chaos 1995, 5, 443. Lázár, A.; Noszticzius, Z.; Försterling, H.-D.; Nagy-Ungvárai, Zs. Physica D 1995, 84, 112 .

(32) Tyson, J. J.; Fife, P. C. J. Chem. Phys. 1980, 73, 2224

(33) Keener, J. P.; Tyson, J. J. Physica D 1986, 21, 307.

(34) Field, R. J.; Noyes, R. M. J. Chem. Phys. 1974, 60, 1877.

(35) Steinbock, O.; Zykov, V.; Müller, S. C. Nature 1993, 366, 322. 